Bull. Austral. Math. Soc.

VOL. 64 (2001) [377-380]

\title{
ON ENTIRE SOLUTIONS OF A CERTAIN TYPE OF NONLINEAR DIFFERENTIAL EQUATION
}

\author{
ChUNG-ChUN YANG
}

In this note, we shall study, via Nevanlinna's value distribution theory, the uniqueness of transcendental entire solutions of the following type of nonlinear differential equation:

$$
L(f(z))-p(z) f^{n}(z)=h(z),
$$

where $L(f)$ denotes a linear differential polynomial in $f$ with polynomials as its coefficients, $p(z)$ a polynomial $(\not \equiv 0), h$ an entire function, and $n$ an integer $\geqslant 3$. We show that if the equation $\left(^{*}\right)$ has a finite order transcendental entire solution, then it must be unique, unless $L(f) \equiv 0$.

\section{INTRODUCTION}

It seems to be quite difficult, in general, to develop a systematic theory or method to show whether or not a nonlinear differential equation has a transcendental entire solution. Nevertheless Nevanlinna's value distribution theory (see, for example [2]), has been utilised to resolve the growth estimates of entire or meromorphic solutions of algebraic differential equations, (see, for example [3]). As far as the author is aware, there is no significant result about the uniqueness of entire or meromorphic solutions of a general class of nonlinear differential equations. This note attempts to introduce such a result, with the goal of stimulating interest among readers, so that more fruitful and profound results on this type of problems will be derived. It is assumed that readers are familiar with the basic results of the Nevanlinna's value distribution theory and its associated notations such as $m(r, f) T(r, f)$, et cetra (see, for example [2]).

\section{MAIN RESULTS}

THEOREM 1. Let $n, k$ be positive integers, with $n \geqslant 3$. Let $p(z)$ be a polynomial $\not \equiv 0, L(f)$ denote a linear differential polynomial in $f, L(f)=q_{0}(z)+p_{0}(z) f+p_{1}(z) f^{\prime}+$

Received 15th January, 2001

The work described in this paper was partially supported by a grant from the Research Grants Council of The Hong Kong Special Administrative Region, China (project no. HKUST 6070/98p).

Copyright Clearance Centre, Inc. Serial-fee code: 0004-9727/01 \$A2.00+0.00. 
$\cdots+p_{k}(z) f^{(k)}$ where $q_{0}(z)$ denotes a polynomial, $p_{i}(i=0,1,2, \cdots, k)$ are polynomials not all identically zero, and $h(z)$ denotes an entire function. Consider the nonlinear differential equation:

$$
L(f)-p(z) f^{n}(z)=h(z)
$$

If the above equation has a transcendental entire solution $f$ of finite order, then either equation (1) has $f$ as its unique transcendental entire solution of finite order or any two such solutions $f_{1}$ and $f_{2}$ must satisfy $L\left(f_{i}\right) \equiv 0(i=1,2)$ and therefore $h(z)$ must assume the form: $-p(z) f_{i}^{n}$.

COROLlaRY. In Theorem 1 , if the entire function $h(z)$ cannot be expressed as $h(z) \equiv q(z) g^{m}(z)$, where $q$ is a polynomial, $g$ an entire function and $m$ an integer $\geqslant 3$, then equation (1) can have at most one transcendental entire solution of finite order.

Note the Tumura-Clunie Theorem (see [2, p.69]) enables us to conclude easily the following result as to the existence of a transcendental entire solution.

THEOREM 2. Suppose the hypotheses of Theorem 1 hold, and assume further that $\bar{N}(r,(1 / h))=o(1) T(r, h)$, then equation (1) can have no transcendental entire solution.

PROOF OF THEOREM 1: Let $f$ be a finite order transcendental entire solution of (1), and let $g$ be any other such transcendental solution. Thus

$$
L(g)-p(z) g^{n}(z)=h(z) .
$$

Assume $f-g \not \equiv 0$. Then it follows from (1) and (2) that

$$
L(f)-L(g)-p(z)\left(f^{n}-g^{n}\right)=0 .
$$

Hence

$$
\frac{L(f)-L(g)}{f-g}=p(z)\left(\frac{f^{n}-g^{n}}{f-g}\right)=p(z) F(z),
$$

where $F(z) \equiv\left(f-\eta_{1} g\right)\left(f-\eta_{2} g\right) \cdots\left(f-\eta_{n-1} g\right)$ and $\eta_{1}, \eta_{2} \cdots, \eta_{n-1}$ are distinct roots, other than 1, of the equation: $z^{n}=1$. Recall the well known "lemma on the logarithmic derivative" (see, for example $[2$, p.34]) and note $f-g$ is of finite order. We have thus from (4) that

$$
O(1) \log r=m\left(r, \frac{L(f)-L(g)}{f-g}\right)=m\left(r, p\left(\frac{f^{n}-g^{n}}{f-g}\right)\right)
$$

Since $F(z) \equiv p\left(\left(f^{n}-g^{n}\right) /(f-g)\right)$ is entire, it follows that $m\left(r, p\left(\left(f^{n}-g^{n}\right) /(f-g)\right)\right)$ $=T\left(r, p\left(\left(f^{n}-g^{n}\right) /(f-g)\right)\right)=O(1) \log r$. Hence $F(z)$ must be a polynomial. If $F$ is identically zero, then one must have $f^{n} \equiv g^{n}$ and $g \equiv \eta_{i} f$, for some integer $i(1 \leqslant i \leqslant$ 
$n-1$ ). Substituting this into (3), we have $L(f)-\eta_{i} L(f)=0$ or $L(f)=0$, which leads to $h(z)=-P(z) f^{n}(z)$ as asserted. Next we deal with the case

$$
F(z)=q(z)
$$

for some polynomial $q(z) \not \equiv 0$. We shall treat the cases when $n \geqslant 4$ and $n=3$ separately. For $n \geqslant 4$, we have from (5) that for $i=1,2, \cdots, n-1$, each $g / f-\eta_{i}$ has only finitely many zeros. By Picard's theorem, it follows that $g / f$ must be a rational function, but then (5) would not hold.

For $n=3$, we have

$$
q(z)=p(z)\left(f-\eta_{1} g\right)\left(f-\eta_{2} g\right),
$$

where $\eta_{i} i=1,2$ are the two distinct primitive roots of $z^{3}-1=0$. It follows that

$$
\begin{aligned}
& f-\eta_{1} g=q_{1} e^{\alpha} \\
& f-\eta_{2} g=q_{2} e^{-\alpha},
\end{aligned}
$$

where $q_{1}, q_{2}$ are polynomials and $\alpha$ is a nonconstant entire function. Solving for $f$ and $g$ from (6), we have

$$
g=\frac{q_{2} e^{-\alpha}-q_{1} e^{\alpha}}{\eta_{1}-\eta_{2}}, \quad f=\frac{\eta_{2} q_{1} e^{\alpha}-\eta_{1} q_{2} e^{-\alpha}}{\eta_{2}-\eta_{1}} .
$$

Subsituting these into (1) and (2) respectively, we have

$$
\begin{aligned}
& L\left(\left(\eta_{2} q_{1} e^{\alpha}-\eta_{1} q_{2} e^{-\alpha}\right) /\left(\eta_{2}-\eta_{1}\right)\right)-p(z)\left(\left(\eta_{2} q_{1} e^{\alpha}-\eta_{1} q_{2} e^{-\alpha}\right) /\left(\eta_{2}-\eta_{1}\right)\right)^{3} \\
& \quad=L\left(\left(q_{2} e^{-\alpha}-q_{1} e^{\alpha}\right) /\left(\eta_{2}-\eta_{1}\right)\right)-p(z)\left(\left(q_{2} e^{-\alpha}-q_{1} e^{\alpha}\right) /\left(\eta_{1}-\eta_{2}\right)\right)^{3} .
\end{aligned}
$$

By applying Borel's type of inequality, see for example [1], we conclude by comparing the coeffcients of the term, say, $e^{3 \alpha}$ in (8) that

$$
\left(\eta_{2} q_{1}\right)^{3}=\left(-q_{1}\right)^{3} .
$$

Hence $\eta_{2}^{3} \equiv-1$, which is impossible. Thus we can conclude, at the very beginning, that $f-g \equiv 0$ or $f \equiv g$. This also completes the proof of the theorem.

REMARKS. (1) It is easily seen from an example that Theorem 1 is not true for $n=2$. For instance, let

$$
\begin{aligned}
f_{1}(z) & =\frac{p(z)-e^{\int p(z) d z}}{2} \\
f_{2}(z) & =\frac{p(z)+e^{\int p(z) d z}}{2}
\end{aligned}
$$

where $p(z)$ is a nonconstant polynomial. Then $f_{1} \not \equiv f_{2}$ but

$$
f_{1}^{2}(z)-f_{1}^{\prime}(z)=f_{2}^{2}(z)-f_{2}^{\prime}(z) .
$$

(2) As an application of the corollary to Theorem 1 one can assert, for instance, the following results. 
(a) That $f(z)=\sin z$ is the unique transcendental entire solution of the nonlinear differential equation:

$$
3 f^{\prime \prime}(z)+4 f^{3}(z)=-\sin 3 z .
$$

(b) Assume that $L(f)$ denotes a linear differential polynomial with constants as the coefficients, $n$ an integer $\geqslant 3$, and $h(z)$ a periodic entire function with period $t$. Then any finite order nonconstant entire solution of the differential equation $L(f)+f^{n}(z)=h(z)$ must be periodic, with a period depending on $t$ and the coefficients of $L(f)$.

\section{REFERENCES}

[1] E. Borel, 'Sur les zeros des fonctions entieres', Acta Math. 20 (1897), 357-396.

[2] W.K. Hayman, Meromorphic functions, Oxford Mathematical Monographs (Clarendon Press, Oxford, 1964).

[3] I. Laine, Nevanlinna theory and complex differential equations (Walter de Grungter, Berlin, New York, 1993).

Department of Mathematics

The Hong Kong University of Science and Technology

Kowloon, Hong Kong, China 